\title{
An in vitro model of innate lymphoid cell function and differentiation
}

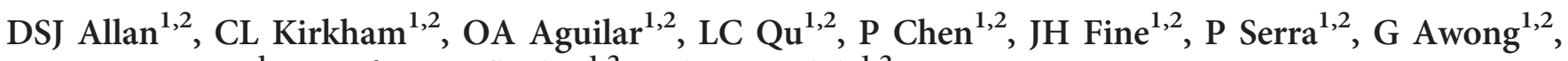
JL Gommerman ${ }^{1}$, JC Zúñiga-Pflücker ${ }^{1,2}$ and JR Carlyle ${ }^{1,2}$

Innate lymphoid cells (ILC) are RAG-independent lymphocytes with important roles in innate immunity, and include group-1 (natural killer (NK) cell, ILC1), group-2 (ILC2), and group-3 (lymphoid tissue inducer (LTi), NCR ${ }^{+}$ILC3) subsets. Group-3 ILC express Roryt, produce interleukin (IL)-22, and are critically important in the normal function of mucosal tissues. Here, we describe a novel model cell line for the study of ILC function and differentiation. The parental MNK cell line, derived from NKR-P1B ${ }^{+}$fetal thymocytes, shows a capacity to differentiate in $\gamma_{c}$ cytokines. One IL-7-responsive subline, designated MNK-3, expresses Roryt and produces high levels of IL-22 in response to IL-23 and IL-1ß stimulation. MNK-3 cells display surface markers and transcript expression characteristic of group-3 ILC, including IL-7R $\alpha$ (CD127), c-kit (CD117), CCR6, Thy1 (CD90), RANK, RANKL, and lymphotoxin (LT $\left.\alpha_{1} \beta_{2}\right)$. Using an in vitro assay of LTi cell activity, MNK-3 cells induce ICAM-1 and VCAM-1 expression on stromal cells in a manner dependent upon LT $\alpha_{1} \beta_{2}$ expression. A second IL-2-responsive subline, MNK-1, expresses several NK cell receptors, perforin and granzymes, and shows some cytotoxic activity. Thus, MNK-1 cells serve as a model of ILC1/NK development and differentiation, whereas MNK-3 cells provide an attractive in vitro system to study the function of ILC3/LTi cells.

\section{INTRODUCTION}

Innate lymphoid cells (ILC) can be defined as cells of lymphoid lineage and morphology that lack functionally rearranged T-cell receptor (TCR) or immunoglobulin (Ig) genes. Recent work has clarified the ILC nomenclature into three subsets. ${ }^{1}$ Group-1 ILC include conventional natural killer (NK) cells that exhibit perforin and granzyme-mediated cytotoxicity against virally infected cells and tumor cells. The signature cytokine secreted by NK cells is interferon (IFN)- $\gamma$. NK cells can be found in the blood, spleen, lymph nodes, liver, uterus, and other tissues. Individuals with NK cell deficiencies are particularly sensitive to Herpesviridae infections. Development of NK cells depends on several transcription factors, including T-bet (encoded by Tbx21), eomesodermin (Eomes), and E4BP4 (Nfil3), as well as signaling via the cytokine receptors CD122 $\left(\mathrm{Il} 2 \mathrm{rb}\right.$ ) and CD132 (common gamma chain, $\left.\gamma_{c}, \mathrm{Il} 2 \mathrm{rg}\right){ }^{2}$ Within the group-1 ILC umbrella, there are also ILC1 cells that produce IFN- $\gamma$ and are distinct from NK cells. ${ }^{1,3,4}$ However, some of these ILC1 cells may derive from ILC3 under the influence of pro-inflammatory stimuli., ${ }^{3,5-7}$

Group-2 ILC are defined by their ability to produce cytokines similar to Th2 cells, especially interleukin (IL)-5 and IL-13. Known variably as innate helper type- 2 cells, nuocytes, or natural helper cells, ILC2 cells ${ }^{1}$ have been identified in lymph node, spleen, liver, lung, intestine, and peritoneal adiposeassociated lymphoid tissue. ${ }^{8}$ Th2 cytokine production by ILC2 is triggered by IL-25 (IL-17E) and IL-33. ${ }^{9}$ Development of ILC2 requires Rora and Gata3. ${ }^{9}$ Deficiencies in ILC2 cells cause severe defects in defense against helminth infections. ${ }^{9}$ ILC2 also have a role in allergic responses, for example, producing IL-5 and IL-13 in response to allergens. ${ }^{9}$

Group-3 ILC are defined by expression of the signature transcription factor, Roryt (encoded by Rorc), which is also necessary for their development. ${ }^{8}$ This group encompasses embryonic lymphoid tissue inducer ( $\mathrm{LTi}$ ) cells and related adult ILC3 cells. ${ }^{1}$ Although distinctions have been made previously, ${ }^{1}$

${ }^{1}$ Department of Immunology, University of Toronto, Toronto, Ontario, Canada and ${ }^{2}$ Sunnybrook Research Institute, Toronto, Ontario, Canada. Correspondence: DSJ Allan or JR Carlyle (david.allan@sri.utoronto.ca or james.carlyle@utoronto.ca) 
here, the term ILC3 will be used to refer inclusively to LTi cells and other Ror $\gamma \mathrm{t}^{+}$ILC. During development, LTi cells colonize the primordial lymph nodes, Peyer's patches, cryptopatches, and isolated lymphoid follicles. LTi cells induce adhesion molecules, chemokines, and the recruitment of other cells to establish the secondary lymphoid organs, via lymphotoxin signaling to mesenchymal organizer cells. ${ }^{10}$ Like Th17 cells, which also express Ror $\gamma$ t, fetal LTi cells produce IL-17A and IL-22. ${ }^{11,12}$ Cells with an LTi-like phenotype are also found in adult intestines and spleen, and similarly have the capacity to make IL-17A and/or IL-22. ${ }^{7,12-14}$ ILC3 also include cells found in mucosal tissues, such as the intestinal lamina propria, that express NKp46 (Ncr1) and produce IL-22 upon stimulation. ${ }^{15-18}$ Also known as NK22 cells, NK cell receptor (NKR)-LTi cells, NCR22 cells, and ILC22, they are now known as $\mathrm{NCR}^{+}$ILC3. ${ }^{1}$ These cells have a limited capacity to produce IL-17A.

In addition to their role in lymphoid tissue development, ILC3 are also indispensible for adult mucosal homeostasis and immune defense. Depletion of ILC3 in vivo causes a loss of containment of commensal bacteria and results in systemic inflammation. ${ }^{19,20}$ ILC3 are also critical in defense against intestinal pathogens such as Citrobacter rodentium, where they are the dominant source of protective IL-22, ${ }^{18,21}$ and condition dendritic cells via lymphotoxin signaling. ${ }^{22}$ ILC3 are also important for clearance of oral fungal infections such as Candida albicans, ${ }^{23}$ and are protective in several models of inflammatory bowel disease. ${ }^{12,24}$ In allogeneic bone marrow transplant models, IL-22-producing ILC3 were reduced during graft-vs.-host disease, leading to intestinal epithelial damage. ${ }^{25}$ In addition, functional ILC3 are required for mucosal production of IgA. ${ }^{26}$

Here, we report a novel cell line, MNK-3, that shares with ILC3 the expression of signature transcription factors, secreted cytokines, cytokine receptors, chemokine receptors, and adhesion molecules. MNK-3 represents a prototypical cell line model of ILC3 that can be expanded in culture to high numbers to study the biology of ILC3 in vitro. In addition, a second subline, MNK-1, serves as a model for the differentiation of other ILC subsets, including IFN- $\gamma$-producing ILC1 and NK cells.

\section{RESULTS \\ MNK cells can differentiate in $\gamma_{c}$ cytokines to generate distinct sublines in vitro}

The MNK cell line was originally generated from sorted NIH-Swiss mouse fetal day-15 NK1.1 $1^{+}$thymocytes that were co-cultured with Bcl2-retrovirus producer cells and subsequently transfected with constructs encoding the SV40 large T antigen (T-Ag) and human c-MYC, then cultured in IL-3, IL6 , IL-7, stem cell factor, and maintained in IL-2 for $\sim 6$ weeks. ${ }^{27}$ The resulting cell line was responsive to the $\gamma_{c}$ cytokines, IL-2, IL-7, and IL-15 in vitro, but lacked cytotoxic activity, and initial characterization was suggestive of a thymic NK precursor. Notably, in this mouse strain, the NK1.1 phenotype is due to expression of the NKR-P1B ${ }^{\mathrm{Sw}}$ receptor (encoded by Klrb1b), in contrast to the well-known NKR-P1C ${ }^{\mathrm{B} 6}$ receptor $(K l r b 1 c) .{ }^{28}$

Interestingly, cultured MNK cells exhibited phenotypic heterogeneity, with some cells expressing additional NKR. To examine this in greater detail, MNK cells were sorted to high purity for lack of NKp46, CD94, NKG2D, DX5, and CD11b expression, then cultured with $\gamma_{c}$ cytokine combinations. Cells cultured in IL-7 cycled slowly and remained negative for these NKR (Figure 1). In contrast, in the presence of IL-2, these NKR were induced de novo on a subset of MNK cells (Figure 1). This differentiation was not due to outgrowth of rare sort contaminants, as clonal analyses revealed the appearance of NKp46, DX5, and CD94 on some cells (data not shown).

Given these results, different sublines were developed. Sorted $\mathrm{NKR}^{-} \mathrm{MNK}$ cells cultured in IL-2 were sequentially sorted for NKp46, CD94, and NKG2D expression, and one resulting subline was designated MNK-1 (Figure 1). A second NKR ${ }^{-}$ subline maintained in IL-7 (then expanded in IL-7 and IL-15) was designated MNK-3 (Figure 1). Genomic DNA PCR revealed integration of SV40 T-Ag and human c-MYC in both MNK-3 and MNK-1 (Supplementary Figure 1 online).

\section{MNK-3 and MNK-1 cells lack markers of most leukocyte lineages, including $T$ cells}

The MNK-3 and MNK-1 cell lines were characterized by flow cytometry. MNK-3 cells did not express markers of T cells

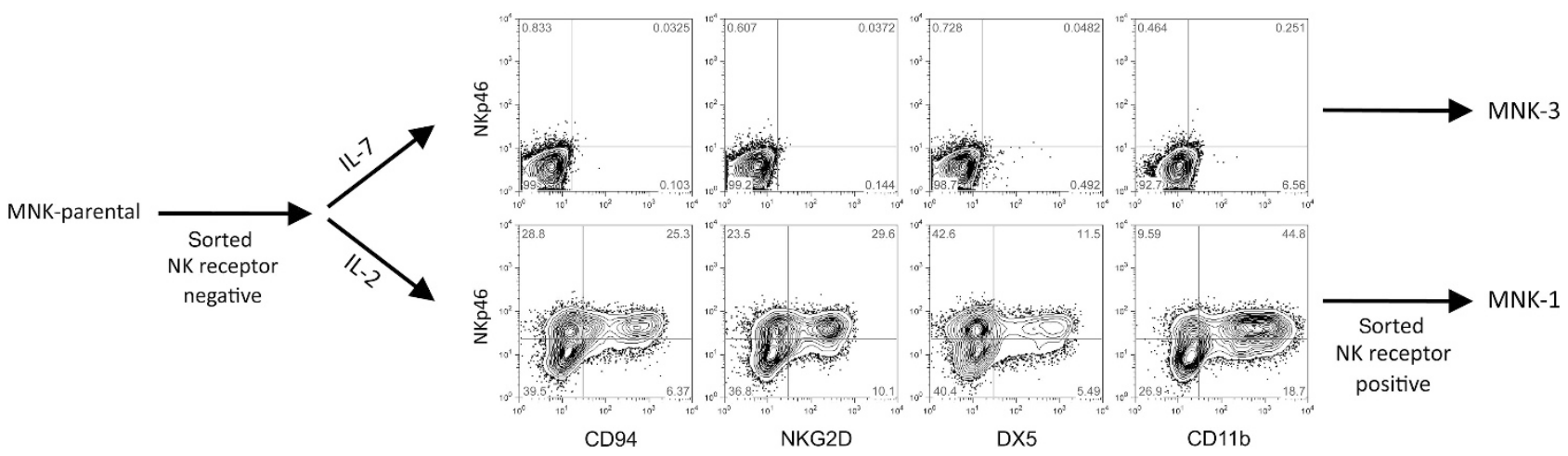

Figure 1 The MNK cell line can differentiate in $\gamma_{c}$ cytokines to generate distinct sublines in vitro. Parental MNK cells were sorted to high purity to be negative for NKp46, CD94, NKG2D, DX5, and CD11b, then cultured for 31 days in interleukin (IL)-7 or IL-2 (each at $10 \mathrm{ng} \mathrm{ml}^{-1}$ ) and analyzed by flow cytometry. Cells maintained in IL-7 were subsequently expanded in IL-7 plus IL-15 and designated MNK-3. Cells cultured in IL-2 were sequentially sorted positive for NKp46, CD94, and NKG2D to generate the MNK-1 subline. NK, natural killer. 
(CD3e, TCR $\gamma \delta$ TCR $\beta, \mathrm{CD} 4, \mathrm{CD} 8 \alpha, \mathrm{CD} 2$, or CD5), B cells (CD19, IgM, CD45R(B220)), dendritic cells (CD11c), macrophages (F4/80), or granulocytes (Gr-1) (Figure 2a). MNK-3 cells also lacked detectable Flt3 (CD135) expression, but did express the normal leukocyte markers, CD11a, major histocompatibility complex (MHC)-I ( $\left.\mathrm{H}-2^{\mathrm{q}}\right)$, and Clr-b (Clec $\left.2 d\right)$ (Figure 2a); notably, the latter functions as a ligand for the NKR-P1B (Klrb1b) inhibitory receptor, ${ }^{29,30}$ which is also expressed at high levels. The second subline, MNK-1, displayed a similar phenotype, with the exception of low-level expression of CD45R and CD11c (Figure 2b), which can be expressed by activated NK cells. ${ }^{31,32}$

The transcriptomes of MNK-3 and MNK-1 cells were profiled by microarray and RNA sequencing (RNA-seq) (along with control syngeneic NIH-Swiss NKp46 ${ }^{+} \mathrm{CD}^{-}$IL-2-lymphokine-activated killer (NK-LAK) cells), then compared with transcriptome data from other cells, including intestinal ILC3 $\left(\mathrm{CD}^{-}{ }^{-} \mathrm{CD} 19^{-}\right.$Roryt-reporter $\left.{ }^{+} \mathrm{NKp} 46^{-}\right),{ }^{33}$ intestinal ILC2 $\left(\mathrm{CD}^{-}{ }^{-} \mathrm{CD} 19^{-} \mathrm{Sca}^{\mathrm{hi}} \mathrm{CD} 25^{+} \mathrm{KLRG1}^{+}\right),{ }^{33}$ splenic NK cells, ${ }^{34}$ and naive splenic $\mathrm{CD} 8{ }^{+} \mathrm{T}$ cells. ${ }^{35}$ Because of their fetal thymic origin, the MNK-3 and MNK-1 cell lines were examined more closely for T-cell-associated gene products. As shown in Figure 2c, MNK-3 cells express negligible transcripts of Cd3e, Cd3d, Cd3g, Cd5, Rag1, Rag2, and Ptcra (pre-TCR $\alpha$ ) (all registering $<1$ sequence read per kilobase per million (r.p.k.m.)). MNK-3 cells also lacked the transcription factors Bcl11b (necessary for T-cell development) and Sox 13 (involved in $\gamma \delta \mathrm{T}$-cell development). Low-level expression of

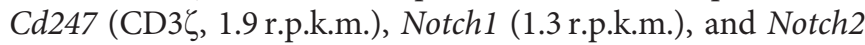
(3.7 r.p.k.m.) was observed in MNK-3 cells (Figure 2c). Parallel triplicate microarray measurements largely confirmed the RNA-seq data (Figure 2d). The expression pattern observed in MNK-1 cells was mostly similar, with the exception of higher levels of $C d 3 g$ and $C d 247$ (Figure 2c,d), which were also observed in NK cells and syngeneic NK-LAK (Figure 2d). High-level expression of many of these T-cellassociated molecules was clearly visible in naive CD8 T cells (Figure 2d). a

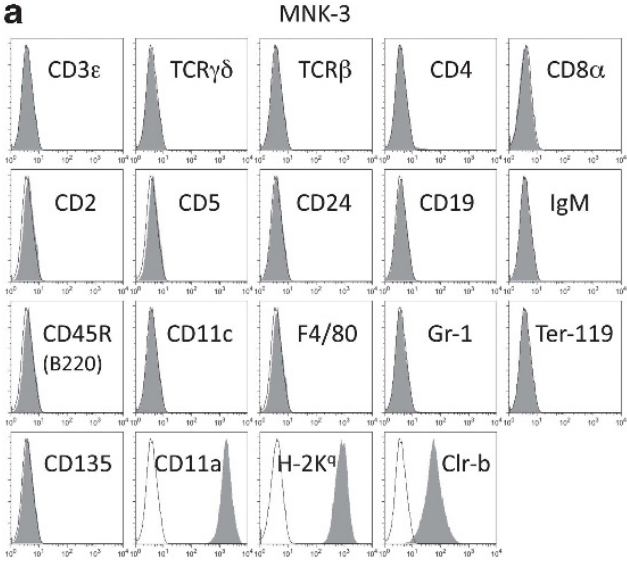

b

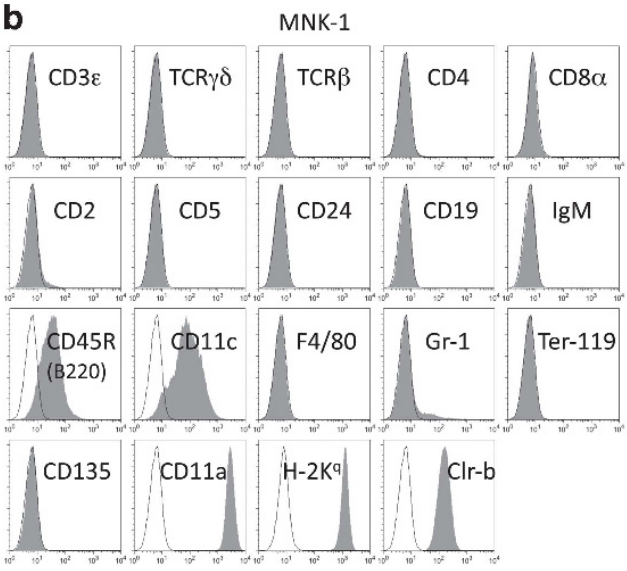

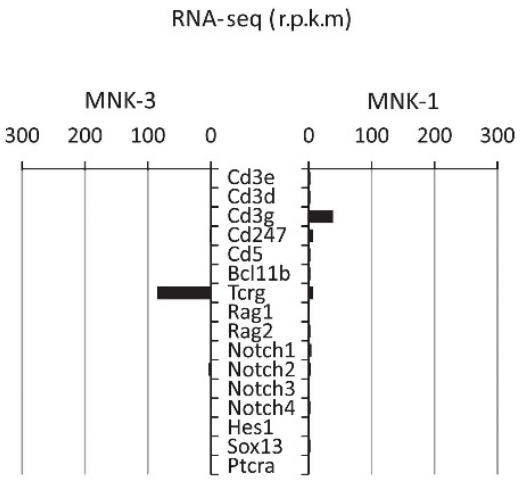

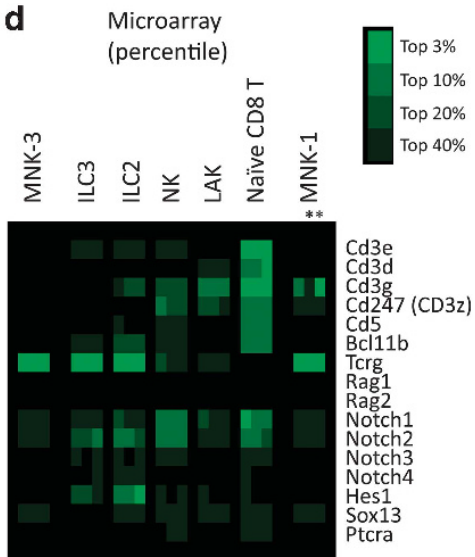

Figure 2 MNK-3 and MNK-1 cells lack markers of most leukocyte lineages, including T cells. (a and $\mathbf{b})$ MNK-3 and MNK-1 sublines were characterized by flow cytometry. The solid-line histograms represent unstained cells, isotype control monoclonal antibody or secondary staining reagent alone. (c) The transcriptomes of MNK-3 and MNK-1 cells were analyzed by whole-transcriptome sequencing. Shown are numbers of sequence reads mapping to each listed gene (reads per kilobase per million (r.p.k.m.)). (d) Transcript expression in MNK-3 and MNK-1 were also examined by Affymetrix microarray. Two related sublines, generated in the same fashion as MNK-1 by independent sorts, were also examined, indicated by asterisks. Depicted are percentile rankings of normalized hybridization intensity for probesets recognizing the listed gene products. Microarray results were contrasted with similar data derived from interleukin (IL)-2-activated splenic NK cells (LAK), ex vivo intestinal Ror $\gamma$ t-reporter ${ }^{+}$ILC3 $3{ }^{33}$ intestinal ILC2, ${ }^{33}$ splenic natural killer (NK) cells, ${ }^{34}$ and naive splenic CD8 T cells. ${ }^{35}$ 
In an exception to this pattern, considerable numbers of MNK-3 and MNK-1 transcriptome reads mapped to the TCR-C $\gamma 1$ (Tcrg-C1) and TCR-C $\beta 1$ (Trbc1) constant regions (Figure 2c; Supplementary Figure 2). However, minimal expression was detected from the TCR-C $\alpha$ or TCR-C $\delta$ constant regions (data not shown). Expression of TCR $\gamma$ was also detected by microarray in both cell lines (Figure 2d), although the cross-reactive probe sets did not distinguish between $\mathrm{C} \gamma$ constant regions. Examination of the MNK-3 and MNK-1 transcriptome sequencing data, PCR-based $5^{\prime}$-RACE reactions, and genomic DNA PCR implied that these were unrearranged germline TCR $\gamma$ and TCR $\beta$ transcripts, which initiated upstream of recombination signal sequences (Supplementary Figure 2). Interestingly, microarray data of ex vivo ILC3 and ILC2 also showed high signals for TCR $\gamma$, but not other T-cell markers (Figure 2d), suggesting that germline TCR transcripts could be a general phenomenon of ILC in vivo. These results suggest that, despite their thymic origin, MNK-3 and MNK-1 do not possess functionally rearranged TCR.

\section{MNK-1 cells express numerous NKR and NK function-associated molecules}

The MNK-3 and MNK-1 lines originated from sorted NK1.1 ${ }^{+}$ (NKR-P1B ${ }^{+}$) cells, and both expressed high levels of Nkrp1b (Klrb1b) transcripts (Figure 3a,b) and surface protein (NK1.1) (Figure 3c,d). Among surface receptors and signaling adaptors associated with NK cell function, MNK-3 appeared to express substantial levels of FcR $\gamma$ (Fcerlg), Tactile (Cd96), CD16 (Fcgr3), NKp46 (Ncr1), Lilrb4, Kir3dl1, and lower levels of Slamf3 (Ly9), Slamf5 (Cd84), EAT-2 (Sh2d1b1), Cd160, and NKR-P1F (Klrb1f) (Figure 3a,b). Notably, most of these transcripts could also be detected in ex vivo ILC3 cells (Figure $3 \mathbf{b}$ ). For example, Nkrp1b (Klrb1b) was expressed at substantial levels in ILC3, ILC2, NK, and IL-2-activated NK cells (LAK) (Figure 3b). Although originally sorted to be negative for NKp46 (Figure 1), low levels of NKp46 protein were observed on a subset of MNK-3 cells, regained after culture with IL-7 and IL-15 (Figure 3c). MNK-3 remained negative or very low for many NKRs (Figure 3a-c), and failed to express perforin (Prf1), granzyme A (Gzma), or IFN- $\gamma$ (Ifng) (Figure 3a,b). However, MNK-3 had substantial transcripts for granzymes B and $\mathrm{C}(\mathrm{Gzmb}, \mathrm{Gzmc})$ (Figure 3a,b), the latter also detected in ex vivo ILC3 at higher levels than resting NK cells (Figure 3b). Thus, MNK-3 cells lack a mature NK cell phenotype, and most NK cell markers expressed in MNK-3 could also be found in ILC3.

In contrast, MNK-1 cells displayed several features characteristic of NK cells. Sorted positive for NKR expression, MNK-1 continued to express NK-related transcripts (Figure 3a,b) and surface molecules (Figure 3d), including NKp46 (Ncr1), NKG2D (Klrk1), CD94 (Klrd1), and CD11b (Itgam). MNK-1 cells also expressed other NKRs and signaling adaptors at much higher levels than MNK-3, including: Ly49E (Klra5; 238-fold), NKG2A (Klrc1; 73-fold), NKG2C (Klrc2; 32-fold), DAP12 (Tyrobp; 31-fold), Klre1 (22-fold), DAP10 (Hcst; 16-fold), DNAM-1 (Cd226; 15-fold), Kir3dl2 (10-fold), NKR-P1A
(Klrb1a; ninefold), Cd160 (sevenfold), NKG2E (Klrc3; sixfold), EAT-2 (Sh2d1b1; fivefold), Tigit (fivefold), 2B4 (Cd244; fourfold), and ERT (Sh2d1b2; fourfold) (Figure 3a,b). Surface NKG2A/ C/E expression was also observed for MNK-1 (Figure 3d); however, surface Ly49 expression was not detected using antiLy49 monoclonal antibodies (mAb) (Figure 3d), possibly due to strain polymorphisms. Strikingly, MNK-1 cells had extremely high levels of transcripts for the cytotoxic effector molecules, perforin (Prf1) and granzymes-A,B,C,D,E,G (Gzma-g) (Figure 3a,b), rivaling $\beta$-actin for transcript abundance, and necessitating a different scale in Figure 3a. Interestingly, the increased NKR and cytotoxic molecules in MNK-1 correlated with higher expression of eomesodermin (Eomes), E4bp4 (Nfil3), and T-bet (Tbx21) transcription factors associated with development of mature NK cells (Figure 3a,b). ${ }^{2}$ Two sublines created in the same manner as MNK-1 by independent sorting were also characterized by microarray, and their results appeared to be similar (Figure 3b).

Notably, the pattern of NK cell-associated transcripts expressed in MNK-1 showed many similarities with those observed for syngeneic NK-LAK cells (columns 5 and 6 in Figure $3 \mathbf{b}$ ). Indeed, when all genes were considered that differed between MNK-3 and MNK-1 (e.g., >10-fold or $>5$-fold in both RNA-seq and microarray analyses), genes with high expression in MNK-1 often displayed elevated levels in LAK cells, whereas genes selectively expressed in MNK-3 were rarely expressed in LAK (Figure 3e; Supplementary Figure 3). Although less than LAK cells, MNK-1 cells showed a low level of cytotoxic activity against classical NK cell target cell lines YAC-1 and RMA/S (Figure 3f). MNK-3 cells did not appear to be cytotoxic (Figure 3f).

\section{MNK- 3 cells express many markers associated with Ror $\gamma \mathrm{t}^{+}$ ILC3}

MNK-3 cells were examined for expression of transcription factors associated with the ILC lineages. Id 2 transcripts were highly expressed in MNK-3 cells, detected by RNA-seq and microarray (Figure 4a,b). Id 2 expression was also seen in ex vivo ILC3, ILC2, NK cells, and syngeneic NK-LAK (Figure 4b), consistent with its necessity for the development of these cell types. ${ }^{8}$ Other transcription factors implicated in the development of multiple ILC lineages, including ILC $3{ }^{36-38}$ such as TCF-1 (Tcf7), Tox, and Gata3, were also expressed by MNK-3 (115, 11, and 3.2 r.p.k.m., respectively) (Figure 4a,b and data not shown). However, PLZF (Zbtb16) transcript, found transiently in ILC precursors ${ }^{39}$ was very low in MNK-3 (1.0 r.p.k.m.) (data not shown).

Strikingly, MNK-3 expressed high levels of Rorc, which defines ILC3 cells and is critical for their formation (Figure $4 a, b)$. Closer RNA-seq analysis revealed that most MNK-3 Rorc transcripts represent the Roryt splice isoform (data not shown). Ror $\gamma \mathrm{t}$ protein was also validated by intracellular flow cytometric analysis of MNK-3 cells (Figure 4c). MNK-3 cells also showed significant expression of Rora (Figure 4a,b). Mice lacking Rora have deficiencies in ILC2 populations, ${ }^{9}$ but microarray data revealed that Rora is expressed in both ILC2 and ILC3 


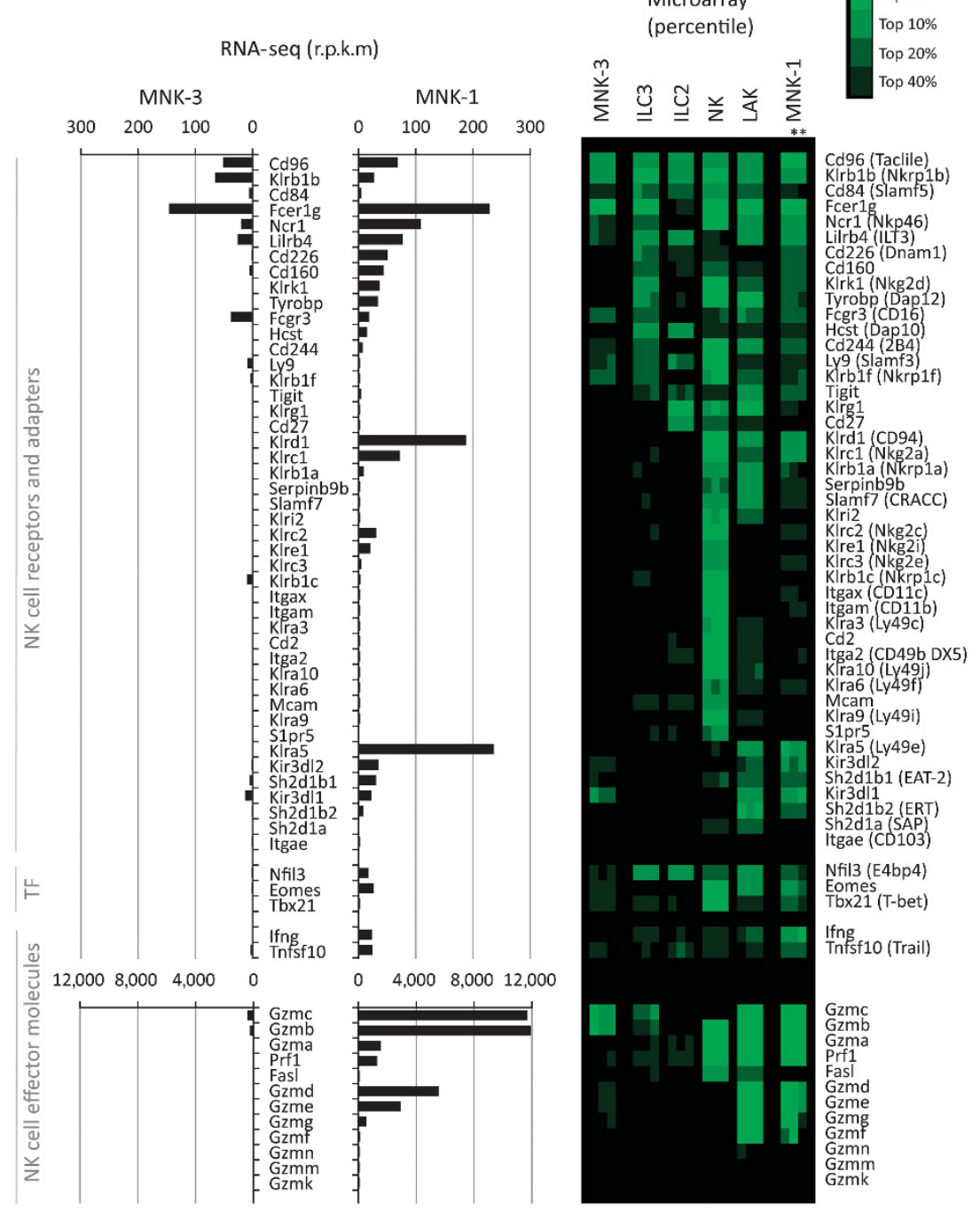

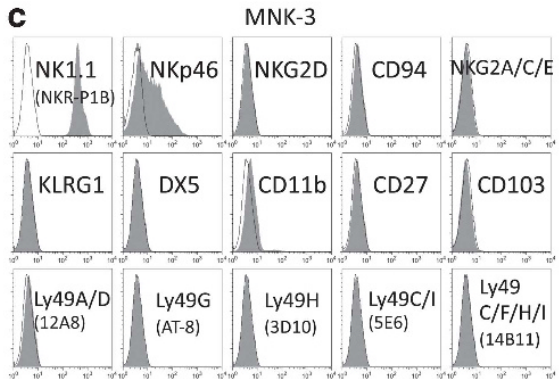

d

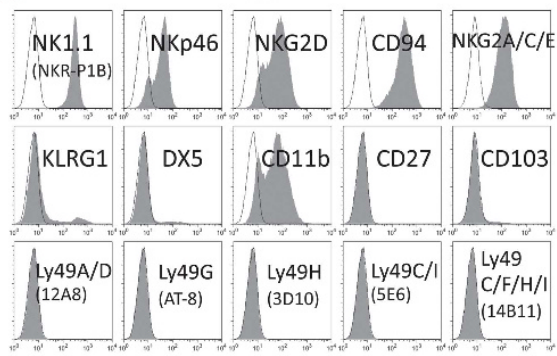

e

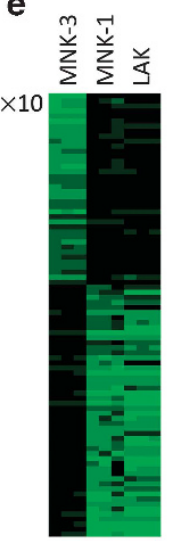

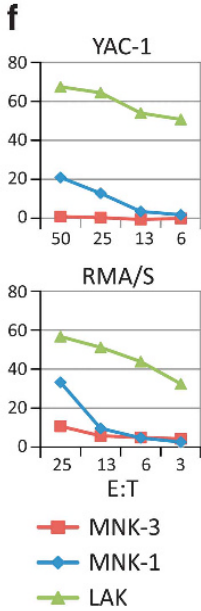

Figure 3 MNK-1 cells display many NK cell receptors (NKR), cytotoxic function molecules, and some cytotoxic activity. MNK-3 and MNK-1 cells were characterized by (a) whole-transcriptome sequencing, (b) Affymetrix microarray, or (c and d) flow cytometry as described in Figure 2. (e) All genes differing between MNK-3 and MNK-1 by the indicated fold change (in both RNA-Seq and microarray analyses) are shown, and contrasted with syngeneic natural killer (NK)-lymphokine-activated killer (LAK) cells. Depicted are percentile rankings of normalized hybridization intensity using the same scale as in b. (f) Cytotoxicity of MNK-3, MNK-1 or LAK cells toward YAC-1 and RMA/S target cells was measured in a ${ }^{51} \mathrm{Cr}$ release assay. Percent specific lysis is shown.

(Figure 4b). Ahr has also been implicated in post-natal proliferation, survival, and function of some ILC3 subsets and the development of isolated lymphoid follicles. ${ }^{40-42}$ MNK3 cells expressed a low but detectable level of $A h r$ transcripts (5.4 r.p.k.m.) (Figure 4a,b). ILC3 may have parallels in gene regulation with Th17 cells. However, among key transcription factors implicated in Th17 regulatory networks, ${ }^{43}$ MNK-3 cells expressed Stat3 and Maf, but lower Batf, and minimal Irf4 (Figure 4a,b).

Given the similarities between MNK-3 and ILC3, we examined molecules associated with ILC3 and ILC2 phenotype and function. Interestingly, MNK-3 expressed a number of transcripts that were highly expressed in multiple ILC subsets, and several transcripts that were specific to ILC3 (Figure 4a,b).
For example, MNK-3 expressed transcripts for cytokine and chemokine receptors found on ILC3 (and/or ILC2) populations, including Il2rg, Il2rb, Il2ra, Il7r, Il23r, Illr1, Il17re, Kit, Cxcr6, Cxcr4, Cxcr5, and Ccr6 (Figure 4a,b). Il2rg and $I L 7 r$ are necessary for efficient development of ILC3 cells, whereas $I l 23 r$ and Illr1 are critical for their response to cytokines. ${ }^{8}$ Expression of cell-surface protein on MNK-3 was demonstrated for CD122 (Il2rb), CD25 (Il2ra), CD127 (Il7r), CD117 (Kit), CXCR4, CXCR5, and CCR6 (Figure 4c).

ILC subsets also express several members of the tumor necrosis factor (TNF)/TNF receptor (TNFR) superfamilies. MNK-3 expressed high levels of Lta and Ltb (Figure 4a,b), prototypical markers of LTi cells, necessary for ILC3 functions in lymphoid organogenesis, dendritic cell-regulation, and 
a

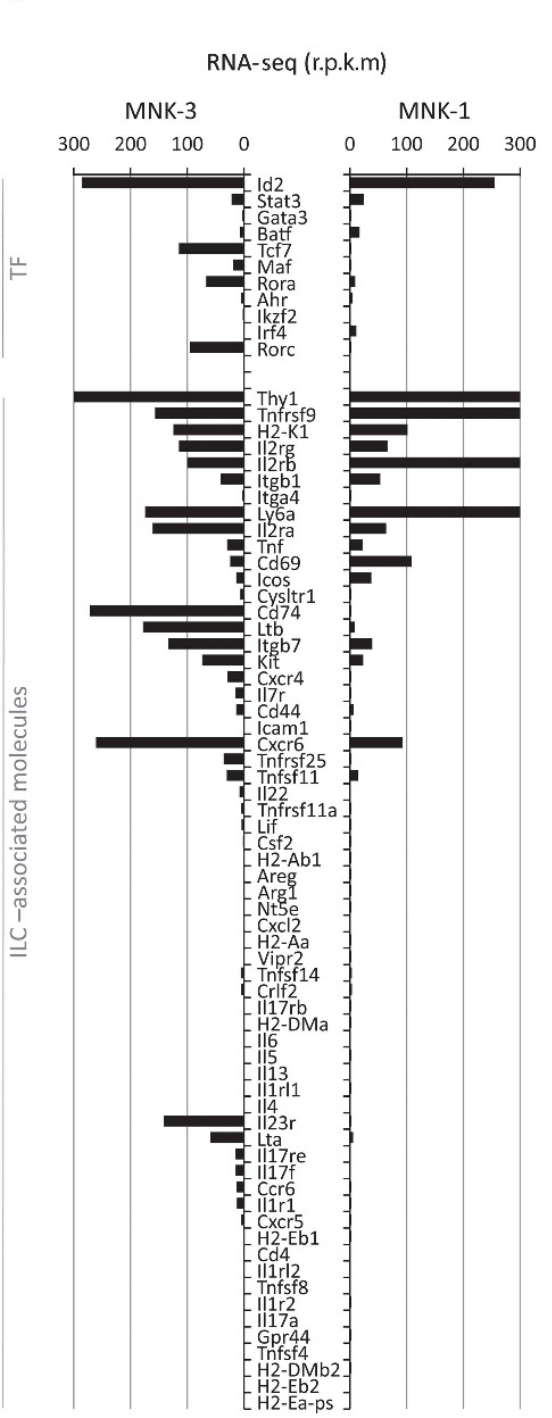

b

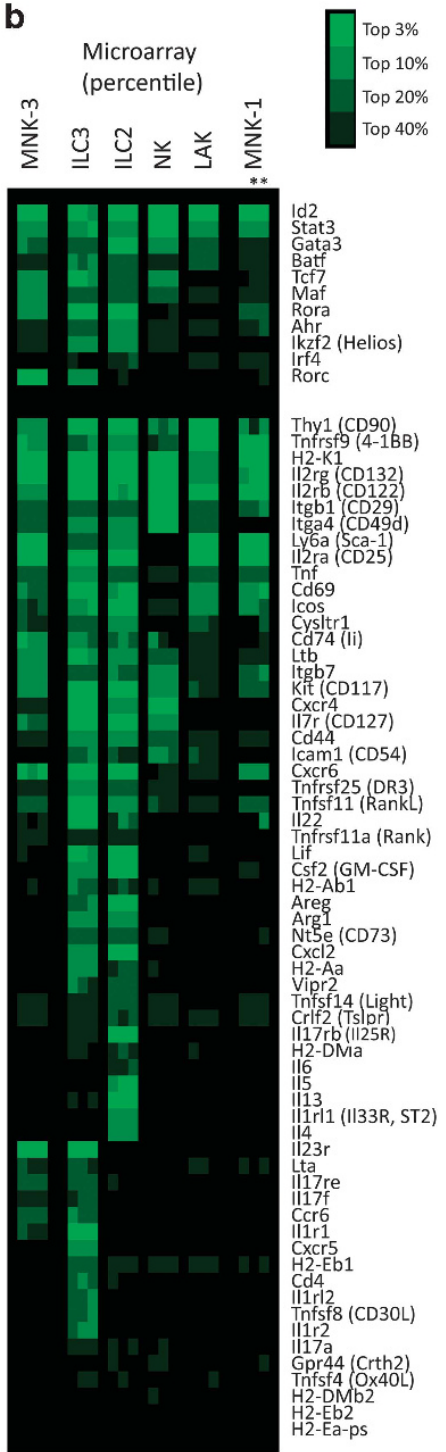

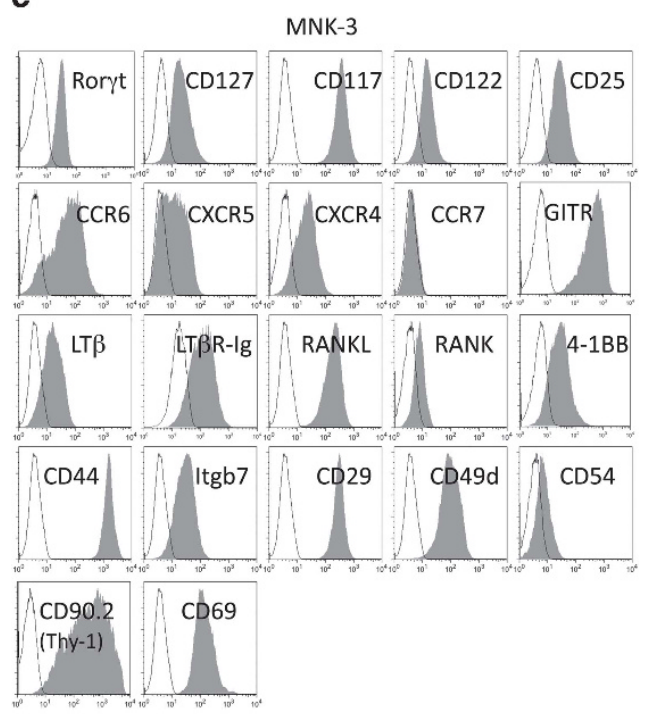

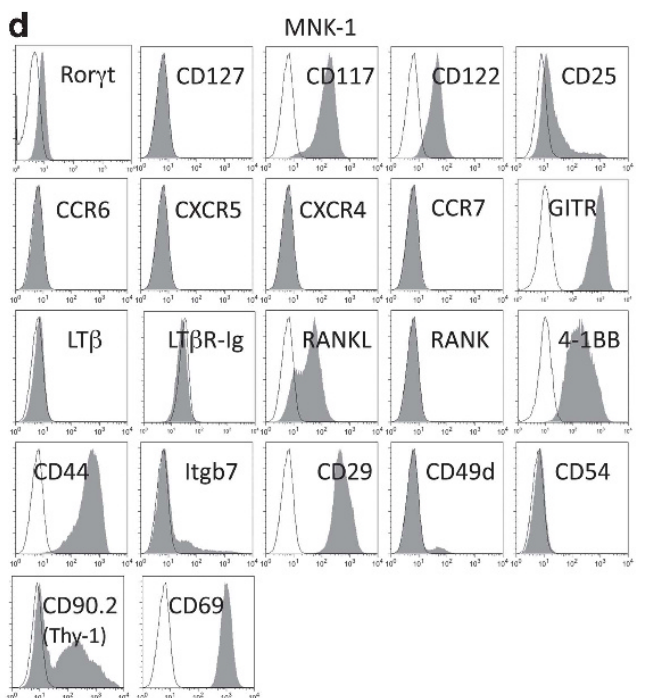

Figure 4 MNK-3 cells express many molecules associated with Ror $\gamma \mathrm{t}^{+}$ILC3. MNK-3 and MNK-1 cells were characterized by (a) whole-transcriptome sequencing, (b) Affymetrix microarray, or (c and d) flow cytometry staining, as described in Figure 2.

control of gut IgA production in vivo. ${ }^{10,22,26}$ Surface LT $\beta$ expression on MNK-3 cells was confirmed by flow cytometry (Figure 4c), and functional binding of LT $\beta$ R-Ig fusion protein to $\mathrm{MNK}-3$ cells likely represents binding to $\mathrm{LT} \alpha_{1} \beta_{2}$ heterotrimers (Figure 4c), because the other LT $\beta$ R ligand, LIGHT (Tnfsf14), was expressed at lower levels in MNK-3 (Figure 4a,b). Like ILC3, MNK-3 also expressed transcripts for 4-1BB (Tnfrsf9), GITR (Tnfrsf18), DR3 (Tnfrsf25), RANKL (Tnfsf11), and RANK (Tnfrsf11a), several of which were confirmed by flow cytometry (Figure 4c).

Characteristic adhesion molecules and surface markers have also been reported on ILC3 and ILC2. Strikingly, MNK-3 showed substantial expression of CD90 (Thy1), integrin- $\beta 7$ (Itgb7), integrin- $\beta 1$ (Itgb1/CD29), integrin- $\alpha 4$ (Itga4/CD49d), CD44, Sca-1 (Ly6a), and CD69 (Figure 4a,b). All of these proteins could be detected on the surface of MNK-3 cells (Figure 4c, data not shown). Notably, MNK-3 did not express transcripts restricted to ILC2 cells, including the signature cytokines, IL-5 and IL-13 (Figure 4b).

On the other hand, the other subline, MNK-1, lacked transcripts for many ILC3-associated molecules found in MNK-3, including Il23r, Il1r1, Il7r, Ccr6, Cxcr5, Cxcr4, Il17re, DR3 (Tnfrsf25), Rank (Tnfrsf11a), and Itga4; MNK-1 also had much reduced levels of $L t a, L t b$, and Itgb7 (Figure 4a,b). Reduction or absence of several of these surface proteins was verified by flow cytometry (Figure 4d). Furthermore, MNK-1 cells lacked Rorc expression and had reduced Rora compared with MNK-3 cells (Figure 4a,b).

\section{MNK-3 produces IL-17A, IL-17F, and substantial IL-22, whereas MNK-1 produces IFN- $\gamma$}

MNK-3 cells were examined for their production of IL-22 and IL-17A, responsible for many in vivo functions of ILC3. Wholetranscriptome sequencing analysis of MNK-3 revealed basal 
expression of IL-22 (7.9 r.p.k.m.), but no detectable IL-17A (Figure 4a,b). Functionally, unstimulated MNK-3 cells secreted a low level of IL-22 (averaging $2 \mathrm{ng} \mathrm{ml}^{-1}$ ) into cell supernatants (Figure 5a). IL-22 production by ILC3 cells can be triggered by exposure to IL-23 or IL-1. ${ }^{8,15}$ Stimulation of MNK-3 cells with IL-1, IL-23, IL-2, or Phorbol 12-myristate 13-acetate (PMA)/ ionomycin caused substantial increases in IL-22 production (averaging 13-48 $\mathrm{ng} \mathrm{m}^{-1}$ ) (Figure 5a). Interestingly, a synergistic effect was observed, whereby simultaneous treatment with IL-1, IL-23, and IL-2 increased IL-22 to remarkable levels (exceeding $200 \mathrm{ng} \mathrm{ml}^{-1}$ ) (Figure 5a). Similar responses in IL-22 production were observed by intracellular flow cytometric analysis (Figure 5b). Small increases in IL-22 production were elicited by the $\gamma_{c}$ cytokines, IL-15 or IL-7, (Supplementary Figure 4) or IL-17C (Figure 5b), which binds to the IL17RE receptor expressed on MNK-3 and ILC3 (Figure $4 \mathbf{a}, \mathbf{b})$. In contrast to MNK-3 results, IL-22 production by MNK-1 was almost undetectable (Figure 5a).

MNK-3 was also capable of producing IL-17A, but only upon potent stimulation, such as synergy of multiple cytokines or upon combination with PMA/ionomycin treatment (Figure 5a). When stimulated with individual cytokines, IL-17A secretion was essentially undetectable (Figure 5a). Similar responses were observed by intracellular staining of MNK-3 (Figure 5b). As with IL-22, MNK-1 did not show any evidence of IL-17A production (Figure 5a).

Transcriptome sequencing data showed basal expression of the related cytokine IL-17F (14.9 r.p.k.m.) in MNK-3, but not in MNK-1 cells (Figure 4a,b). IL-17B, IL-17C, IL-17D, and IL-25 (IL-17E) were all very low or absent (data not shown). Intracellular staining of MNK-3 confirmed significant production of IL-17F, with a pattern similar to that observed for IL-22: IL-1 alone, or cytokine combinations including IL-1, had the greatest capacity to induce IL-17F expression (Figure 5b). ILC3 are reported to be the primary source of granulocytemacrophage colony-stimulating factor in the gut. ${ }^{44}$ Similarly, MNK-3 cells secreted granulocyte-macrophage colony-stimulating factor at levels much higher than MNK-1, again triggered by IL-1 (Figure 5a). The production of additional cytokines and chemokines by MNK-3 and MNK-1 were also investigated (Supplementary Figure 5). Strikingly, IFN- $\gamma$, the signature ILC1/NK cytokine, was only produced by MNK-3 cells using multiple cytokine stimuli in combination with PMA/ ionomycin (Figure 5a,b). In contrast, the other subline, MNK1 , produced basal IFN- $\gamma$ levels (averaging $0.3 \mathrm{ng} \mathrm{m}^{-1}$ ) and very high levels of IFN- $\gamma$ upon stimulation with IL-2 and/or PMA/ionomycin (Figure 5a).

ILC2 cells are potent producers of Th2 cytokines, including IL-5 and IL-13, upon stimulation with IL-25 and/or IL-33. ${ }^{9}$ MNK-3 produced low levels of IL-4 and IL- 6 upon stimulation with IL-23 and IL-1, respectively (Figure 5c). However, incubations with IL-25 and IL-33 had no effect (with or without IL-2 and/or PMA/ionomycin) (Figure 5c). This result is consistent with the transcript data that showed minimal expression of IL-25 receptor (Ill7rb) and IL-33 receptor (Il1rl1) in MNK-3 (Figure 4a,b).
In summary, MNK-3 produces cytokines characteristic of ILC3, using the same stimuli known to trigger ex vivo ILC3. In contrast, MNK-1 cells produce primarily IFN- $\gamma$, the signature cytokine of NK cells and ILC1.

\section{MNK-3 possesses LTi-like activity in vitro}

During embryonic development, ILC3 cells of the LTi subcategory provide lymphotoxin-mediated signals to mesenchymal organizer cells. This leads to upregulation of adhesion molecules and chemokines in the mesenchymal cells, and supports lymphoid organogenesis. ${ }^{10}$ While characterizing human fetal LTi cells, Cupedo $e a^{11} l^{11}$ utilized an in vitro assay: co-culture of human LTi cells with human fetal mesenchymal stem cells that led to upregulation of ICAM-1 and VCAM-1 on the mesenchymal cells, which was used as a readout of LTi activity. A variation of this assay was performed using MNK-3 and the murine OP9 stromal cell line reported to share features of bona fide mesenchymal stem cells. ${ }^{45}$ Specifically, we used a variant of the OP9 line that expresses low ICAM-1, but considerable VCAM-1 on the surface (Figure 6). Strikingly, co-culture with MNK-3 cells caused marked induction of ICAM-1 expression on OP9 cells and a further increase in VCAM-1 levels (Figure 6). Importantly, these effects were lymphotoxin dependent, as they could be abrogated using blocking LT $\beta$ $\mathrm{mAb}$ (Figure 6) or soluble LT $\beta \mathrm{R}-\mathrm{Ig}$ fusion protein (data not shown). Thus, MNK-3 cells display LTi-like activity in vitro upon incubation with mesenchymal stromal cells, similar to the influence of ex vivo LTi cells.

MNK-3 cells engraft in vivo and can be genetically modified To further investigate the utility of MNK-3 cells as a model, we examined the capacity to genetically modify these cells. MNK-3 cells were readily modified by transient transfection or lentiviral transduction using green fluorescent protein reporter vectors $^{46}$ (Supplementary Figure 6). In addition, upon adoptive transfer into immunodeficient mice, MNK-3 cells engrafted and proliferated with a doubling time of $\sim 7$ days. At early time points, MNK-3 cells appeared home to sites of primary hematopoiesis (e.g., bone marrow), then localize chiefly to gastrointestinal and uterine tissues (Supplementary Figure 7).

\section{DISCUSSION}

MNK-3 cells exhibit many features characteristic of ILC3, which include several overlapping and related cell subsets. The prototypical ILC3 subset is the fetal LTi cell. LTi cells are the first hematopoietic cells to colonize the lymph node anlagen, and initiate secondary lymphoid organ formation, including the development of Peyer's patches, cryptopatches, and isolated lymphoid follicles in the intestine. ${ }^{10}$ Fetal LTi cells were originally characterized by Mebius et al..$^{47}$ as $\mathrm{CD} 3{ }^{-} \mathrm{CD} 4{ }^{+}$cells that express LT $\beta, \alpha 4 \beta 7$ integrin, CXCR5, CD45, CD90 (Thy1), CD25 ( 75\%), CD127, CD132, CD44, MHC-I, MHC-II ( $~ 50 \%$ ), and ICAM-1. With the exception of CD4, MHC-II, and ICAM-1 expression, this phenotype is recapitulated by MNK-3. However, subsequent studies reported the existence of fetal LTi cells lacking CD4, in numbers comparable to $\mathrm{CD} 4{ }^{+}$ 


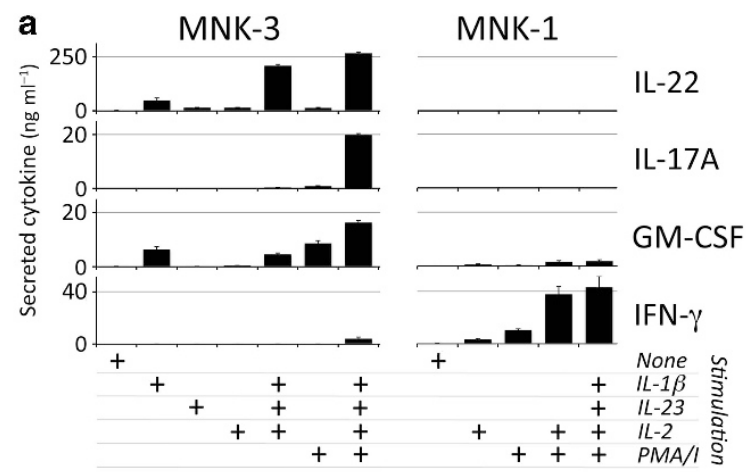

b

MNK-3
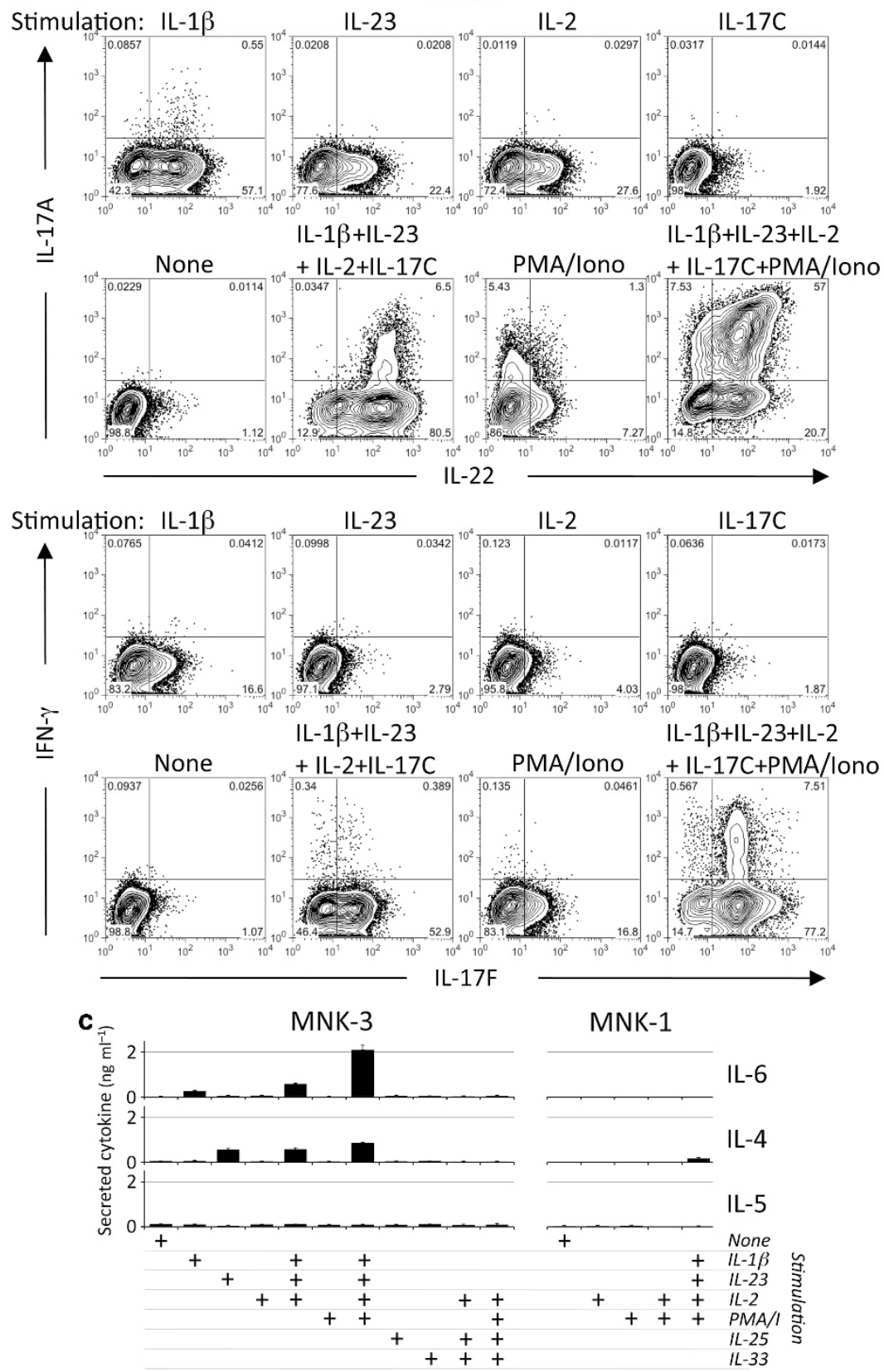

MNK-1

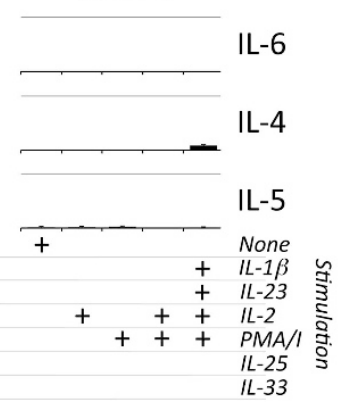

Figure 5 MNK-3 cells produce substantial interleukin (IL)-22 in response to IL-1 $\beta$ and IL-23, whereas MNK-1 cells secrete interferon (IFN)- $\gamma$. (a and c) MNK-3 or MNK-1 cells were stimulated as indicated for $\sim 20 \mathrm{~h}$. Secretion of the listed cytokines was detected by multiplex bead immunoassay ( $n=3$ ). (b) Intracellular flow cytometry analysis was performed on MNK-3 cells stimulated for $\sim 7 \mathrm{~h}$. Results are typical of three or four similar experiments. 


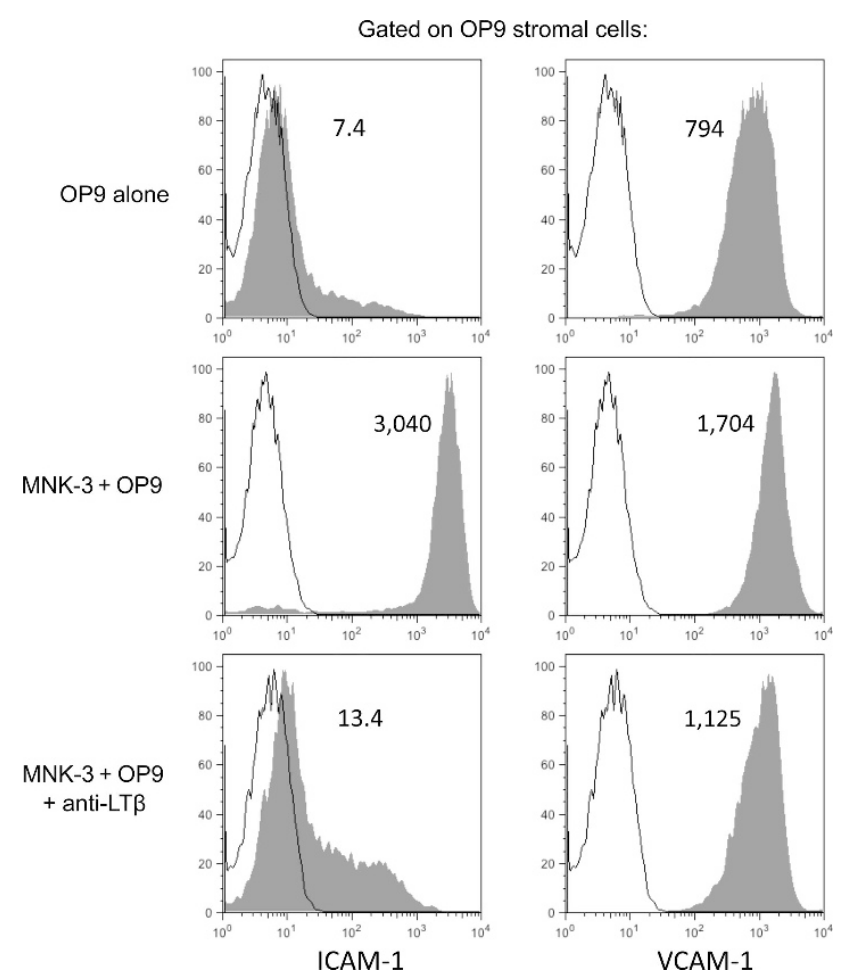

Figure 6 MNK-3 cells display LTi-like activity in vitro. A green fluorescent protein (GFP)-transduced variant of the OP9 stromal cell line that expresses low ICAM-1 was cultured alone or co-cultured with MNK-3 cells for 3 days, before analysis by flow cytometry. Plots are gated on the $\mathrm{GFP}^{+}$OP9 cells. Effects of lymphotoxin were blocked using an anti-LT $\beta$ monoclonal antibody. Numbers depict median flourescent intensity.

$\mathrm{LTi}^{14,48}$ perhaps more closely resembling MNK-3. It is possible that expression of MHC-II and ICAM-1 may depend on certain stimuli or interactions in vivo.

Several molecules are important in the development and function of fetal LTi cells. Mice deficient in Id2 or Rorc lack LTi cells, and consequently have no lymph nodes or Peyer's patches. ${ }^{8,49}$ In addition, mice deficient in Cxcr5, Ltb, Lta, RANK, RANKL, $I l 7 r$, or Il2rg lack lymph nodes and/or Peyer's patches. ${ }^{49}$ Strikingly, MNK-3 expresses all of these molecules. Similar to fetal LTi, MNK-3 cells were able to deliver $\mathrm{LT} \alpha_{1} \beta_{2}$ signals to stromal organizer cells, leading to induction of ICAM-1 and VCAM-1 expression (Figure 6).

Cells with a LTi-like phenotype can also be detected in neonatal and adult animals. ILC3 cells in small intestine include $\mathrm{CCR}^{+}{ }^{+}$and $\mathrm{CCR}^{-}{ }^{-}$subsets, with CCR6 ${ }^{+}$ILC3 representing the majority during embryonic and neonatal development, declining in proportion thereafter. ${ }^{7,14}$ MNK-3 cells express CCR6, consistent with their embryonic origin. LTi cells and $\mathrm{CCR}^{+}{ }^{+}$LTi-like cells can produce considerable levels of IL-22 and IL-17A, ${ }^{7,11-14}$ similar to MNK-3 cells (Figure 5).

The adult thymus contains ILC3 with a phenotype remarkably similar to $\mathrm{MNK}-3\left(\mathrm{CD}_{4}{ }^{+} \mathrm{CD} 27^{+} \mathrm{CD}^{-} \mathrm{CD}{ }^{-}\right.$ $\left.\mathrm{CCR6}^{+} \mathrm{RANKL}^{+} \mathrm{IL}^{+} 23 \mathrm{R}^{+}\right) .{ }^{50}$ These cells facilitate thymic regeneration after total body irradiation in an IL-22-dependent manner. ${ }^{50}$ Ror $\gamma \mathrm{t}^{+}$ILC3 with an LTi cell phenotype are also found in embryonic day-14 thymus, ${ }^{51}$ and thymic LTi express RANKL and induce Aire expression on medullary thymic epithelial cells via RANK signaling. ${ }^{52}$ MNK-3 expresses high levels of RANKL (Figure 4). As MNK-3 originated from day-15 fetal thymocytes, we hypothesize that MNK-3 may represent an immortalized fetal thymic Ror $\gamma \mathrm{t}^{+}$LTi cell.

Despite its fetal origin, MNK-3 also shares similarities with adult ILC3, including LTi-like and $\mathrm{NCR}^{+}$ILC3. Similar to $\mathrm{NCR}^{+}$ILC3, MNK-3 expresses NKp46 on a subset of cells (Figure 3). Although sorted to lack NKp46, levels of NKp46 on MNK-3 increased during culture with IL-7 + IL-15 (data not shown). Ex vivo $\mathrm{NCR}^{+}$ILC3 are potent producers of IL-22, but lack IL-17A production. ${ }^{12,15-18}$ Similarly, MNK-3 cells produce basal and inducible IL-22 upon exposure to IL-23 or IL-1 (Figure 5). High-level IL-17A production was tightly regulated in MNK-3, requiring stimulation with both cytokines and mitogens (Figure 5). Therefore, MNK-3 serves as a model to study IL-17A induction in ILC3. It is possible that in vivo adult ILC3 also require a synergy of stimuli to initiate inflammatory IL-17A production. Attractive candidates include IL-23 and IL-1 coupled with signals from cell surface receptors. ${ }^{53}$ Understanding the triggers of IL-17A production has disease relevance, as pathological IL-17A-producing ILC3 exist in inflammatory contexts such as Helicobacter hepaticus infection. ${ }^{54}$ The phenotype of these colitis-exacerbating cells $\left(\mathrm{CD}_{0}{ }^{+} \mathrm{CD}_{2} 27^{+} \mathrm{CD}_{4}{ }^{+} \mathrm{CCR}^{+}{ }^{+} \mathrm{CD} 25^{+}\right.$Ror $_{\mathrm{t}}{ }^{+} \mathrm{LT} \alpha{ }^{+}$

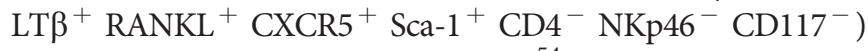
is again strikingly similar to $\mathrm{MNK}-3 .{ }^{54}$

MNK-3 also expressed the NKR-P1B (Klrb1b) inhibitory receptor that recognizes the widely expressed Clr-b (Clec $2 d)$ ligand. ${ }^{29,30}$ Interestingly, ex vivo Ror $\gamma \mathrm{t}^{+}$ILC3 and ILC2 also show considerable microarray signals for NKR-P1B (Figure 3), suggesting these subsets are regulated by 'self Clr-b, similar to NK cells. However, it is not clear whether NKR-P1B is expressed on all ILC3 and ILC2 or subsets in vivo. Comparisons of genes most differentially expressed between MNK-3 and MNK-1 provide additional predictions of novel factors likely to be important in ILC function and development (Supplementary Figure 3).

ILC3 function in a remarkable number of contexts, including development of lymphoid tissues, ${ }^{10}$ upregulation of Aire in thymic epithelial cells, ${ }^{52}$ mucosal homeostasis and containment of commensal bacteria, ${ }^{19}$ defense against bacterial and fungal infections, ${ }^{15,18,21,23}$ inhibition of inflammatory bowel disease pathology, ${ }^{12,24}$ thymic regeneration following irradiation, ${ }^{50}$ graft-vs.-host disease pathology following bone marrow transplantation, ${ }^{25}$ regulation of anti-commensal T-cell responses, ${ }^{20}$ and control of mucosal IgA production. ${ }^{26}$ Therefore, MNK-3 may serve as an attractive model for the function of ILC3 in many physiological and pathological situations. The capacity to genetically modify MNK-3 cells with ease (Supplementary Figure 6) will facilitate studies involving gene overexpression, knockdown, or CRISPR/Cas9-mediated deletion in vitro or in vivo (Supplementary Figure 7).

Interestingly, the parental MNK cell line may also serve as a model of ILC differentiation. When cultured in IL-2, MNK cells 
induced several NKRs de novo, including NKp46, CD94, and NKG2D (Figure 1). Resulting MNK-1 cells produced significant quantities of IFN- $\gamma$ (Figure 5). It is not yet clear whether this represents clonal differentiation of ILC3-like Ror $\gamma \mathrm{t}^{+}$cells into ILC1-like cells under the influence of IL-2, or differentiation of bipotential progenitor cells from a heterogeneous MNK population. Precedents for either possibility exist. First, it has been reported that ILC3 cells exhibit some plasticity and can differentiate into ILC1-like cells producing IFN- $\gamma$. When sorted human NKp $44^{+} \mathrm{CCR}^{+}$ILC3 from tonsils were cultured with IL- 2 for $8-15$ days, a substantial proportion $(>20 \%)$ acquired the ability to produce IFN- $\gamma .^{6}$ Strikingly, culture of these cells in IL-1, IL-7, and IL-23 caused the majority to express CD94. ${ }^{6}$ Upon adoptive transfer of murine ILC3 into immunodeficient mice, a proportion of cells induced NKp46 while losing Roryt expression. ${ }^{5}$ In addition, genetic fate-mapping experiments implied that some Ror $\gamma \mathrm{t}^{+}$ ILC3 could downregulate Ror $\gamma \mathrm{t}$, correlating with increased IFN- $\gamma$ production. ${ }^{5}$ This process was inhibited by IL-7 signaling, ${ }^{5}$ mirroring our results with MNK (Figure 1). Nonetheless, CCR6 ${ }^{+}$LTi-like adult ILC3 may not represent precursors of $\mathrm{NCR}^{+}$ILC3 in vivo. It has been suggested that CCR $^{+}{ }^{+}$and CCR6 ${ }^{-}$ILC3 are distinct lineages, with only the latter capable of acquiring NKp46 expression and IFN- $\gamma$ production in a process dependent upon T-bet. ${ }^{7,55}$ Interestingly, IFN- $\gamma$-producing MNK-1 cells showed higher levels of T-bet (Tbx21) (2.4 r.p.k.m.) compared with MNK-3 (0.4 r.p.k.m.). It is possible that chronic inflammatory stimulation may allow upregulation of T-bet in CCR6 ${ }^{+}$ ILC in vivo. MNK differentiation may model the ILC3 to ILC1 transition.

Notably, MNK-1 cells cultured in IL-2 expressed not only NKp46 and IFN- $\gamma$, but also several other NKR and cytotoxic molecules. Therefore, they may resemble bona fide NK cells or other distinct ILC1 subsets (such as intraepithelial IFN- $\gamma$ producing ILC1). ${ }^{4}$ There are few murine NK cell/ILC1 models, highlighting the utility of MNK-1. Both NK cells and Ror $\gamma \mathrm{t}^{+}$ ILC3 can develop from common lymphoid progenitors ( Lin $^{-}$ $\mathrm{CD} 117^{\text {int }} \mathrm{CD} 127^{+}$). In particular, when a fetal liver common lymphoid progenitor subset expressing integrin $\alpha 4 \beta 7$ was cultured with IL-7, stem cell factor, and feeder cells, predominantly NK/ILC1 cells (NKp46 ${ }^{+}$Ror $^{-} \mathrm{t}^{-}$) and ILC3 (expressing Id2, Rorc, LT $\beta$, IL23R, IL-22, CXCR5, and CCR6) were generated. ${ }^{51,56}$ The latter phenotype closely resembles MNK-3, whereas the former resembles MNK-1. Similar $\alpha 4 \beta 7^{+}$ common lymphoid progenitors were found in E14 fetal thymus. ${ }^{51}$ Recently, progenitor cells with PLZF ${ }^{\text {high }} \mathrm{CD} 117^{+}$ $\mathrm{CD} 127^{+} \alpha 4 \beta 7^{+}$phenotype have been shown to give rise to several ILC lineages. ${ }^{39}$ These populations may overlap with bipotent fetal thymic TNK progenitors expressing CD117, CD127, and NKR-P1B, similar to MNK-3. ${ }^{57,58}$ Therefore, during the genesis of the MNK cell line, it is possible that one of these progenitor cells was immortalized and retained the ability to differentiate into both NK cells and ILC3. This differentiation of MNK may mirror the wave of NK cell development observed in the thymus during embryonic development. ${ }^{58}$

\section{METHODS}

Cell culture. MNK-3 and MNK-1 lines were cultured in Dulbecco's modified Eagle's medium-high glucose medium with $10 \%$ fetal bovine serum, 2 mm GlutaMAX, 1 mm sodium pyruvate, $55 \mu \mathrm{m}$ 2-mercaptoethanol, $10 \mathrm{~mm}$ HEPES, $50 \mu \mathrm{g} \mathrm{ml}^{-1}$ gentamicin, $100 \mathrm{U} \mathrm{ml}^{-1}$ penicillin, and $100 \mu \mathrm{g} \mathrm{ml}^{-1}$ streptomycin (all from HyClone (South Logan, UT) or Gibco Life Technologies (Grand Island, NY). MNK-3 cells were maintained in $10 \mathrm{ng} \mathrm{ml}^{-1}$ mouse IL-7 with or without $10 \mathrm{ng} \mathrm{ml}^{-1}$ mouse IL-15. MNK-1 were cultured with $10 \mathrm{ng} \mathrm{ml}^{-1}$ mouse IL-2 or 2,000 $\mathrm{U} \mathrm{ml}^{-1}$ human IL-2 (hIL-2) (Proleukin, Novartis Pharmaceuticals Canada, Dorval, QC, Canada). Murine cytokines were obtained from Peprotech (Rocky Hill, NJ), with the exceptions of IL-23, IL-17C (eBioscience, San Diego, CA), and IL-25 (BioLegend, San Diego, CA).

Whole-transcriptome sequencing and microarray analyses. LAK cells were generated from NIH-Swiss mouse splenocytes by culture with $2,000 \mathrm{U} \mathrm{ml}^{-1}$ hIL-2 for 4 days, then flow cytometry sorting for $\mathrm{NKp} 46^{+} \mathrm{CD}^{-}$cells, followed by two additional days of culture with hIL-2. MNK-3 cells (cultured with IL-7 and IL-15), MNK-1 cells (cultured with hIL-2), and LAK cells were detached with 2 mM EDTA and resuspended immediately in RNAprotect Cell (Qiagen, Hilden, Germany). Also examined were two related lines similar to MNK-1, but generated by independent sorting. RNA was isolated by RNeasy Plus (Qiagen). Seventy-five and $35 \mathrm{bp}$ paired-end SOLiD 5500XL whole-transcriptome sequencing was performed by the Sunnybrook Research Institute Genomics Core Facility (Toronto, ON, Canada) using ribosomal RNA-depleted samples (RiboMinus Eukaryote Kit for RNA-Seq, Life Technologies, Carlsbad, CA). Sequence data were mapped to the Mus musculus genome (build 37.2) with Geospiza GeneSifter Analysis Edition (Geospiza Perkin Elmer, Seattle, WA) using the Bowtie aligner (settings: -fr -C -S -X 10000 -chunkmbs 256). Gene expression was presented as r.p.k.m. (mapped reads per million/ gene size in kilobases). Fold changes were calculated using r.p.k.m. values incremented by 1 to eliminate undefined values. Microarray samples were prepared using the Ambion WT kit (Life Technologies) before hybridization to Mouse Gene 1.0 ST Arrays (Affymetrix, Santa Clara, CA) by the The Centre for Applied Genomics (The Hospital for Sick Children, Toronto, ON, Canada) Microarray CEL files were normalized with Partek Genomics Suite version 6.6 (Copyright, Partek Inc., St. Louis, MO) using the RMA algorithm (with default settings). For comparison, CEL files of experiments utilizing the same GeneChip to analyze small intestine ILC3 $\left(\mathrm{CD}^{-} \mathrm{CD}^{-}{ }^{-}\right.$Rorc reporter ${ }^{+}$ $\left.\mathrm{NKp} 46^{-}\right)^{33}$ and ILC2 (CD3 ${ }^{-} \mathrm{CD}_{19}{ }^{-} \mathrm{Sca}^{\mathrm{hi}} \mathrm{KLRG}^{+}{ }^{+} \mathrm{CD} 25^{+}$ $\left.\mathrm{Id} 2^{+}\right)^{33}$ were downloaded (ArrayExpress E-MEXP-3743; L1-3, N1-3) and normalized in the same fashion. CEL files from the ImmGen project using the identical GeneChips examining splenic NK cells, ${ }^{34}$ and naive splenic CD8 T cells ${ }^{35}$ (GEO: GSM538315, GSM538316, GSM538317, GSM538415, GSM538416, GSM538417) were similarly normalized. To facilitate comparisons between samples processed at independent centers, for each sample, probe sets were percentile ranked by normalized hybridization intensity values. Probe sets in the top $40 \%$ of values are colored green in Figures 2-4. For ImmGen data sets, this is close to the calculated threshold for $95 \%$ probability of expression (www.immgen.org). For MNK-3 and MNK-1 wholetranscriptome sequencing data sets, a similar threshold (top $40 \%$ of expression values) equates to $\sim 1.8$ and 1.1 r.p.k.m., respectively.

Flow cytometry and cytokine production. Fluorochrome-conjugated or biotinylated $\mathrm{mAb}$ were obtained from eBioScience, Becton Dickinson, Pharmingen (Franklin Lakes, NJ), BioLegend or purified in-house. Anti-LT $\beta$ mAb (BBF6) and LT $\beta$ R-Ig were provided by Biogen Idec (Cambridge, MA). For intracellular cytokine analysis after cytokine stimulation, monensin was added after $\sim 2 \mathrm{~h}$, followed by fixation with Cytofix/Cytoperm (Becton Dickinson Pharmingen) after $\sim 5$ additional hours and staining with anti-IL-22 (1H8PWSR), IL-17A (eBio17B7), IL-17F (eBio18F10), or IFN- $\gamma$ (XMG1.2). 
Intracellular staining was also performed after Cytofix/Cytoperm treatment with anti-Ror $\gamma \mathrm{t}$ (AJKJS-9). Cytokine secretion was measured by mouse IL-22 Simplex and Th1/Th2 10plex FlowCytomix cytokine bead array (eBioscience), or by Eve Technologies (Calgary, AB, Canada) Mouse 32plex Discovery Assay.

Cytotoxicity assays. ${ }^{51}$ Chromium release assays were performed using MNK-3, MNK-1, or unsorted C57BL/6 LAK cells (generated by culturing splenocytes with hIL-2 for 6-7 days).

SUPPLEMENTARY MATERIAL is linked to the online version of the paper at http://www.nature.com/mi

\section{ACKNOWLEDGMENTS}

We thank Dr Aruz Mesci, Miho Tanaka, Jaehun Ma and Dr Michele Anderson for assistance and helpful discussions, and Dr Maria Ciofani for critical reading of the manuscript. We are grateful to Korosh Kianizad for providing mouse fetal thymic tissue, Dr Yutaka Amemiya, Dr Arun Seth and Sunnybrook Research Institute Genomics Core Facility for next-generation sequencing, and Gisele Knowles, Courtney McIntosh, Arian Khandani for flow cytometry sorting. We graciously thank Biogen Idec for kindly supplying anti-LT $\beta$ mAb BBF6 and LT $\beta R$-Ig, Dr Tania Watts for providing $m A b$ recognizing 4-1BB and GITR, and Dr Sam Kung for the Cppt-2E vector. This work was supported by funds from the Canadian Institutes for Health Research (CIHR 106491, to J.R.C.) and startup funds from the Sunnybrook Research Institute. J.R.C. was supported by a New Investigator Award from the CIHR and an Investigator in the Pathogenesis of Infectious Disease Award from the Burroughs Wellcome Fund, USA.

\section{DISCLOSURE}

The authors declared no conflict of interest.

(c) 2015 Society for Mucosal Immunology

\section{REFERENCES}

1. Spits, H. et al. Innate lymphoid cells-a proposal for uniform nomenclature. Nat. Rev. Immunol. 13, 145-149 (2013).

2. Vosshenrich, C.A. \& Di Santo, J.P. Developmental programming of natural killer and innate lymphoid cells. Curr. Opin. Immunol. 25, 130-138 (2013).

3. Bernink, J.H. et al. Human type 1 innate lymphoid cells accumulate in inflamed mucosal tissues. Nat. Immunol. 14, 221-229 (2013).

4. Fuchs, A. et al. Intraepithelial type 1 innate lymphoid cells are a unique subset of IL-12- and IL-15-responsive IFN-gamma-producing cells. Immunity 38, 769-781 (2013).

5. Vonarbourg, C. et al. Regulated expression of nuclear receptor RORgammat confers distinct functional fates to NK cell receptor-expressing RORgammat(+) innate lymphocytes. Immunity 33, 736-751 (2010).

6. Cella, M., Otero, K. \& Colonna, M. Expansion of human NK-22 cells with IL7, IL-2, and IL-1 beta reveals intrinsic functional plasticity. Proc. Natl. Acad. Sci. USA 107, 10961-10966 (2010).

7. Klose, C.S. et al. A T-bet gradient controls the fate and function of CCR6RORgammat + innate lymphoid cells. Nature 494, 261-265 (2013).

8. Spits, H. \& Cupedo, T. Innate lymphoid cells: emerging insights in development, lineage relationships, and function. Annu. Rev. Immunol. 30, 647-675 (2012).

9. Walker, J.A. \& McKenzie, A.N. Development and function of group 2 innate lymphoid cells. Curr. Opin. Immunol. 25, 148-155 (2013).

10. Finke, D. Fate and function of lymphoid tissue inducer cells. Curr. Opin. Immunol. 17, 144-150 (2005).

11. Cupedo, T. et al. Human fetal lymphoid tissue-inducer cells are interleukin 17-producing precursors to RORC + CD127 + natural killer-like cells. Nat. Immunol. 10, 66-74 (2009).

12. Sawa, S. et al. RORgammat + innate lymphoid cells regulate intestinal homeostasis by integrating negative signals from the symbiotic microbiota. Nat. Immunol. 12, 320-326 (2011).

13. Takatori, H. et al. Lymphoid tissue inducer-like cells are an innate source of IL-17 and IL-22. J. Exp. Med. 206, 35-41 (2009).
14. Sawa, S. et al. Lineage relationship analysis of RORgammat + innate lymphoid cells. Science 330, 665-669 (2010).

15. Cella, M. et al. A human natural killer cell subset provides an innate source of IL-22 for mucosal immunity. Nature 457, 722-725 (2009).

16. Luci, C. et al. Influence of the transcription factor RORgammat on the development of NKp46 + cell populations in gut and skin. Nat. Immunol. 10, 75-82 (2009).

17. Sanos, S.L. et al. RORgammat and commensal microflora are required for the differentiation of mucosal interleukin 22-producing NKp46 + cells. Nat. Immunol. 10, 83-91 (2009).

18. Satoh-Takayama, N. et al. Microbial flora drives interleukin 22 production in intestinal NKp46 + cells that provide innate mucosal immune defense. Immunity 29, 958-970 (2008).

19. Sonnenberg, G.F. et al. Innate lymphoid cells promote anatomical containment of lymphoid-resident commensal bacteria. Science 336, 1321-1325 (2012).

20. Hepworth, M.R. et al. Innate lymphoid cells regulate CD4+ T-cell responses to intestinal commensal bacteria. Nature 498, 113-117 (2013).

21. Sonnenberg, G.F., Monticelli, L.A., Elloso, M.M., Fouser, L.A. \& Artis, D. $\mathrm{CD} 4(+)$ lymphoid tissue-inducer cells promote innate immunity in the gut. Immunity 34, 122-134 (2011).

22. Tumanov, A.V. et al. Lymphotoxin controls the IL-22 protection pathway in gut innate lymphoid cells during mucosal pathogen challenge. Cell Host Microbe. 10, 44-53 (2011).

23. Gladiator, A., Wangler, N., Trautwein-Weidner, K. \& LeibundGut-Landmann, S. Cutting edge: IL-17-secreting innate lymphoid cells are essential for host defense against fungal infection. J. Immunol. 190, 521-525 (2013).

24. Zenewicz, L.A. et al. Innate and adaptive interleukin-22 protects mice from inflammatory bowel disease. Immunity 29, 947-957 (2008).

25. Hanash, A.M. et al. Interleukin-22 protects intestinal stem cells from immune-mediated tissue damage and regulates sensitivity to graft versus host disease. Immunity 37, 339-350 (2012).

26. Kruglov, A.A. etal. Nonredundant function of soluble LTalpha3 produced by innate lymphoid cells in intestinal homeostasis. Science 342, 1243-1246 (2013).

27. Carlyle, J.R. et al. Mouse NKR-P1B, a novel NK1.1 antigen with inhibitory function. J. Immunol. 162, 5917-5923 (1999).

28. Carlyle, J.R. et al. Molecular and genetic basis for strain-dependent NK1.1 alloreactivity of mouse NK cells. J. Immunol. 176, 7511-7524 (2006).

29. lizuka, K., Naidenko, O.V., Plougastel, B.F., Fremont, D.H. \& Yokoyama, W.M. Genetically linked C-type lectin-related ligands for the NKRP1 family of natural killer cell receptors. Nat. Immunol. 4, 801-807 (2003).

30. Carlyle, J.R. et al. Missing self-recognition of Ocil/Clr-b by inhibitory NKRP1 natural killer cell receptors. Proc. Natl. Acad. Sci. USA 101, 3527-3532 (2004).

31. Vosshenrich, C.A. et al. CD11cloB220 + interferon-producing killer dendritic cells are activated natural killer cells. J. Exp. Med. 204, 2569-2578 (2007).

32. Blasius, A.L., Barchet, W., Cella, M. \& Colonna, M. Development and function of murine B220 + CD11c + NK1.1 + cells identify them as a subset of NK cells. J. Exp. Med. 204, 2561-2568 (2007).

33. Hoyler, T. et al. The transcription factor GATA-3 controls cell fate and maintenance of type 2 innate lymphoid cells. Immunity 37, 634-648 (2012).

34. Bezman, N.A. et al. Molecular definition of the identity and activation of natural killer cells. Nat. Immunol. 13, 1000-1009 (2012).

35. Heng, T.S. \& Painter, M.W. Immunological Genome Project, Consortium. The Immunological Genome Project: networks of gene expression in immune cells. Nat. Immunol. 9, 1091-1094 (2008).

36. Tanriver, Y. \& Diefenbach, A. Transcription factors controlling development and function of innate lymphoid cells. Int. Immunol. 26, 119-128 (2014).

37. Mielke, L.A. et al. TCF-1 controls ILC2 and NKp46 + RORgammat + innate lymphocyte differentiation and protection in intestinal inflammation. J. Immunol. 191, 4383-4391 (2013).

38. Serafini, N. et al. Gata3 drives development of RORgammat + group 3 innate lymphoid cells. J. Exp. Med. 211, 199-208 (2014).

39. Constantinides, M.G., McDonald, B.D., Verhoef, P.A. \& Bendelac, A. A committed precursor to innate lymphoid cells. Nature 508, 397-401 (2014).

40. Qiu, J. et al. The aryl hydrocarbon receptor regulates gut immunity through modulation of innate lymphoid cells. Immunity 36, 92-104 (2012). 
41. Kiss, E.A. et al. Natural aryl hydrocarbon receptor ligands control organogenesis of intestinal lymphoid follicles. Science 334, 1561-1565 (2011).

42. Lee, J.S. et al. AHR drives the development of gut ILC22 cells and postnatal lymphoid tissues via pathways dependent on and independent of Notch. Nat. Immunol. 13, 144-151 (2012).

43. Ciofani, M. et al. A validated regulatory network for Th17 cell specification. Cell 151, 289-303 (2012)

44. Mortha, A. et al. Microbiota-dependent crosstalk between macrophages and ILC3 promotes intestinal homeostasis. Science 343, 1249288 (2014).

45. Gao, J. et al. Characterization of OP9 as authentic mesenchymal stem cell line. J. Genet. Genomics 37, 475-482 (2010).

46. Tran, J. \& Kung, S.K. Lentiviral vectors mediate stable and efficient gene delivery into primary murine natural killer cells. Mol. Ther. 15, 1331-1339 (2007).

47. Mebius, R.E., Rennert, P. \& Weissman, I.L. Developing lymph nodes collect CD4 + CD3- LTbeta + cells that can differentiate to APC, NK cells, and follicular cells but not T or B cells. Immunity 7, 493-504 (1997).

48. Kim, M.Y. et al. Heterogeneity of lymphoid tissue inducer cell populations present in embryonic and adult mouse lymphoid tissues. Immunology 124 , 166-174 (2008).

49. Randall, T.D., Carragher, D.M. \& Rangel-Moreno, J. Development of secondary lymphoid organs. Annu. Rev. Immunol. 26, 627-650 (2008).

50. Dudakov, J.A. et al. Interleukin-22 drives endogenous thymic regeneration in mice. Science 336, 91-95 (2012).
51. Cherrier, M., Sawa, S. \& Eberl, G. Notch, Id2, and RORgammat sequentially orchestrate the fetal development of lymphoid tissue inducer cells. J. Exp. Med. 209, 729-740 (2012).

52. Rossi, S.W. et al. RANK signals from $\mathrm{CD} 4(+) 3(-)$ inducer cells regulate development of Aire-expressing epithelial cells in the thymic medulla. J. Exp. Med. 204, 1267-1272 (2007).

53. Glatzer, T. et al. RORgammat( + ) innate lymphoid cells acquire a proinflammatory program upon engagement of the activating receptor NKp44. Immunity 38, 1223-1235 (2013).

54. Buonocore, S. et al. Innate lymphoid cells drive interleukin-23-dependent innate intestinal pathology. Nature 464, 1371-1375 (2010).

55. Rankin, L.C. et al. The transcription factor T-bet is essential for the development of NKp46 + innate lymphocytes via the Notch pathway. Nat. Immunol. 14, 389-395 (2013).

56. Possot, C. et al. Notch signaling is necessary for adult, but not fetal, development of RORgammat(+ ) innate lymphoid cells. Nat. Immunol. 12 , 949-958 (2011).

57. Carlyle, J.R. et al. Identification of a novel developmental stage marking lineage commitment of progenitor thymocytes. J. Exp. Med. 186, 173-182 (1997).

58. Carlyle, J.R. \& Zuniga-Pflucker, J.C. Developmental origins of thymusderived natural killer cells. In Natural Killer Cells: Basic Science and Clinical Application (Lotze, M.T. \& Thomson, A.W., eds) 25-40 (Academic Press, London, UK, 2009). 